\title{
Don't Use English Dev: On the Zero-Shot Cross-Lingual Evaluation of Contextual Embeddings
}

\author{
Phillip Keung Yichao Lu Julian Salazar Vikas Bhardwaj \\ Amazon \\ $\{$ keung, yichaolu, julsal,vikab\}@amazon.com
}

\begin{abstract}
Multilingual contextual embeddings have demonstrated state-of-the-art performance in zero-shot cross-lingual transfer learning, where multilingual BERT is fine-tuned on one source language and evaluated on a different target language. However, published results for mBERT zero-shot accuracy vary as much as 17 points on the MLDoc classification task across four papers. We show that the standard practice of using English dev accuracy for model selection in the zero-shot setting makes it difficult to obtain reproducible results on the MLDoc and XNLI tasks. English dev accuracy is often uncorrelated (or even anti-correlated) with target language accuracy, and zero-shot performance varies greatly at different points in the same fine-tuning run and between different fine-tuning runs. These reproducibility issues are also present for other tasks with different pre-trained embeddings (e.g., MLQA with XLM-R). We recommend providing oracle scores alongside zero-shot results: still fine-tune using English data, but choose a checkpoint with the target dev set. Reporting this upper bound makes results more consistent by avoiding arbitrarily bad checkpoints.
\end{abstract}

\section{Introduction}

Zero-shot and zero-resource cross-lingual NLP has seen significant progress in recent years. The discovery of cross-lingual structure in word embedding spaces culminated in the work of Lample et al. (2018b), which showed that unsupervised word translation via adversarial mappings is competitive with supervised techniques. Concurrent work in machine translation also showed that it is possible to achieve non-trivial BLEU scores without any bitext (Artetxe et al., 2018; Lample et al., 2018a). Self-supervised multilingual contextual embeddings like mBERT (Devlin et al., 2019) and
XLM (Conneau and Lample, 2019) have shown remarkably strong performance on cross-lingual named entity recognition, text classification, dependency parsing, and other tasks (e.g., Pires et al., 2019; Keung et al., 2019; Wu and Dredze, 2019).

Much of this recent work has demonstrated that mBERT performs very well on zero-shot tasks, superseding prior techniques as the baseline for zeroshot cross-lingual transfer learning. By zero-shot, we mean that no parallel text or labeled data from the target language was used during model training, fine-tuning, or hyperparameter search. In this setting, models are trained on labeled (usually English) text and tested on target (non-English) text. Standard practice prohibits the use of target language data for model selection; the final model is chosen using the English dev set only.

However, we find that zero-shot mBERT results can vary greatly. We present 4 published baselines for zero-shot cross-lingual document classification on MLDoc (Schwenk and Li, 2018) in Table 1:

\begin{tabular}{lccccc}
\hline MLDoc & En & Fr & $\mathrm{Ja}$ & $\mathrm{Ru}$ & $\mathrm{Zh}$ \\
\hline Eisenschlos et al. (2019) & 93.2 & $\mathbf{8 3 . 0}$ & 64.6 & 71.6 & 66.2 \\
Dong and de Melo (2019) & $\mathbf{9 4 . 2}$ & 80.0 & $\mathbf{7 3 . 2}$ & 70.7 & 75.4 \\
Keung et al. (2019) & $\mathbf{9 4 . 2}$ & 73.5 & 72.8 & $\mathbf{7 3 . 7}$ & 76.0 \\
Wu and Dredze (2019) & $\mathbf{9 4 . 2}$ & 72.6 & 56.5 & $\mathbf{7 3 . 7}$ & $\mathbf{7 6 . 9}$ \\
\hline$\Delta$ & 1.0 & 10.4 & 16.7 & 3.0 & 10.7 \\
\hline
\end{tabular}

Table 1: Published mBERT baselines on zero-shot cross-lingual text classification on 4 of 7 target languages of MLDoc. On non-English languages, we see disagreements of up to $17 \%$ (absolute) per column. No paper consistently outperforms the results of another.

Even though the authors report English accuracies which are basically identical, their target language performances are very different. Given that each experiment starts with the same pre-trained mBERT model and MLDoc dataset, it is clear that these cross-lingual results are not reproducible. 


\begin{tabular}{lcccccccc}
\hline MLDoc & Dev En & De & Es & Fr & It & Ja & Ru & Zh \\
\hline Max & 97.7 & 87.6 & 82.3 & 78.4 & 69.2 & 67.7 & 71.7 & 70.2 \\
Min & 96.8 & 81.7 & 77.7 & 63.4 & 63.2 & 60.6 & 64.5 & 62.6 \\
$\Delta$ & 0.9 & $\mathbf{5 . 9}$ & $\mathbf{4 . 6}$ & $\mathbf{1 5 . 0}$ & $\mathbf{6 . 0}$ & $\mathbf{7 . 1}$ & $\mathbf{7 . 2}$ & $\mathbf{7 . 6}$ \\
\hline
\end{tabular}

\begin{tabular}{lccccccccccccccc}
\hline XNLI & Dev En & $\mathrm{Ar}$ & $\mathrm{Bg}$ & $\mathrm{De}$ & $\mathrm{El}$ & $\mathrm{Es}$ & $\mathrm{Fr}$ & $\mathrm{Hi}$ & $\mathrm{Ru}$ & $\mathrm{Sw}$ & $\mathrm{Th}$ & $\mathrm{Tr}$ & $\mathrm{Ur}$ & $\mathrm{Vi}$ & $\mathrm{Zh}$ \\
\hline Max & 82.8 & 66.0 & 69.7 & 71.8 & 67.6 & 75.8 & 74.6 & 61.7 & 69.6 & 50.9 & 55.3 & 61.9 & 60.2 & 71.3 & 71.3 \\
Min & 81.9 & 63.3 & 66.8 & 70.0 & 64.8 & 73.6 & 72.9 & 58.4 & 67.3 & 47.8 & 51.0 & 60.3 & 56.3 & 69.2 & 68.6 \\
$\Delta$ & 0.9 & $\mathbf{2 . 7}$ & $\mathbf{2 . 9}$ & 1.8 & $\mathbf{2 . 8}$ & 2.2 & 1.7 & $\mathbf{3 . 3}$ & 2.3 & 3.1 & $\mathbf{4 . 3}$ & 1.6 & $\mathbf{3 . 9}$ & 2.1 & $\mathbf{2 . 7}$ \\
\hline
\end{tabular}

Table 2: Zero-shot accuracies over 10 independent mBERT fine-tuning experiments on MLDoc and XNLI. For each run, we computed the zero-shot accuracies using the checkpoint with the best En dev performance. We show the minimum and maximum accuracy for each task across the 10 experiments. En dev scores are within $0.9 \%$ of each other, but non-En test scores vary by much more depending on the language/corpus. $\Delta \geq 2.5 \%$ are bolded.

For the listed target languages, the highest accuracy is up to 3 points better than the next best and up to 17 points better than the worst. We investigate this reproducibility issue in both MLDoc and XNLI (Conneau et al., 2018), which is another major dataset for evaluating cross-lingual transfer. The variations in published baselines on these and other datasets are summarized in Sec. 6.

In Section 3, we show that the final zero-shot accuracies between and within independent mBERT training runs are highly variable. Variations over different random seeds are similar in magnitude to those in Table 1, with variation due to checkpoint selection using English dev being a significant underlying cause. In Section 4, we find that in many cases, English (En) dev accuracy is not predictive of target language performance. In fact, for some languages, En dev performance is actually anti-correlated with target language accuracy.

Poor correlation between En dev and target test accuracy, combined with high variance between independent runs, means that published zero-shot accuracies are somewhat arbitrary. In addition to zero-shot results, we recommend reporting oracle results, where one still fine-tunes using En data, but uses the target dev set for checkpoint selection.

\section{Experimental setup}

We use cased mBERT (110M parameters) for all of our experiments. We illustrate the reproducibility issues in zero-shot cross-lingual transfer through the document classification task in MLDoc ${ }^{1}$ and the natural language inference task in XNLI. ${ }^{2}$ For the MLDoc and XNLI experiments, we used their full En training sets (10k and 393k examples, re-

\footnotetext{
${ }^{1}$ https://github.com/facebookresearch/MLDoc

${ }^{2}$ https://cims.nyu.edu//sbowman/xnli/
}

spectively). Unless stated otherwise, we use their En development sets ( $1 \mathrm{k}$ and $2.5 \mathrm{k}$ examples) and non-En test sets (4k and 5k examples). When finetuning mBERT, we used a constant learning rate of $2 \times 10^{-6}$ with a batch size of 32 for MLDoc, and a constant learning rate of $2 \times 10^{-5}$ with a batch size of 32 for XNLI. Checkpoints were saved at regular intervals: one checkpoint for every $2 \%$ of the training corpus processed. Models were trained until convergence based on En dev accuracy. We used the mBERT implementation in GluonNLP (Guo et al., 2020) and an AWS p3.8xlarge instance for our MLDoc and XNLI runs, which completed in $\sim 3$ and $\sim 12$ hours respectively on a single NVIDIA V100 GPU.

\section{Between-run and within-run variation}

Running mBERT fine-tuning under different random seeds yields highly variable results, similar to what we observed in Table 1. Previous work on evaluation over random initializations (e.g., Melis et al., 2018) reported relatively small effects on the test metric (e.g., \pm 1 point on En F1 for NER). However, we observed much larger variations in zero-shot accuracy on MLDoc and XNLI.

First, we observed significant variation between independent runs (Table 2). We ran mBERT finetuning with different random seeds, and for each run, selected the checkpoint with the best En dev performance. The best checkpoint from each run gave very different zero-shot results, varying as much as $15.0 \%$ absolute in French (Fr) in MLDoc and $4.3 \%$ in Thai (Th) in XNLI.

Second, we observed significant variation within each run, which we illustrate in Figure 1. En dev accuracy reaches a stable plateau as mBERT finetuning proceeds; however, zero-shot Spanish (Es) 

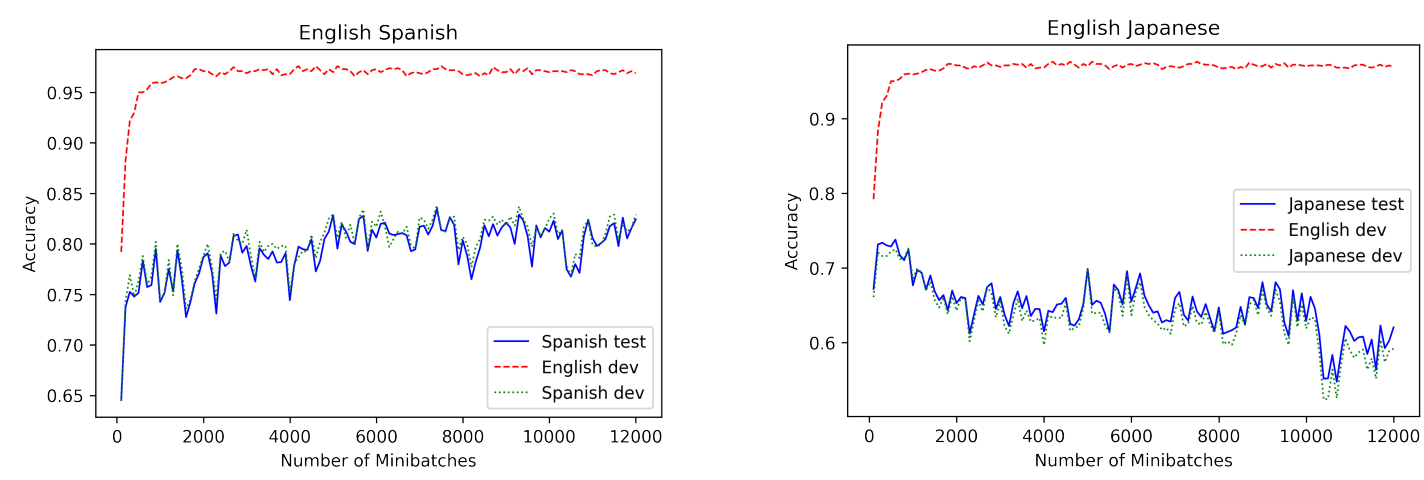

Figure 1: En and non-En MLDoc accuracies over a single mBERT fine-tuning run. English and Spanish (59\% directional agreement) accuracy tend to increase together, whereas English and Japanese (42\% directional agreement) accuracy move in opposite directions. Directional agreement below 50\% indicates improvement on the English set at the expense of non-English languages.

\begin{tabular}{llcccccccc}
\hline \multirow{3}{*}{ MLDoc } & Dev lang. & En & De & Es & Fr & It & Ja & Ru & Zh \\
\cline { 2 - 9 } & English & 0.84 & 0.57 & 0.59 & 0.57 & 0.56 & $\mathbf{0 . 4 2}$ & 0.55 & 0.50 \\
& Target & - & 0.97 & 0.92 & 0.97 & 0.95 & 0.98 & 0.90 & 0.95 \\
\hline
\end{tabular}

\begin{tabular}{llccccccccccccccc}
\hline \multirow{2}{*}{ XNLI I } & Dev lang. & $\mathrm{En}$ & $\mathrm{Ar}$ & $\mathrm{Bg}$ & $\mathrm{De}$ & $\mathrm{El}$ & $\mathrm{Es}$ & $\mathrm{Fr}$ & $\mathrm{Hi}$ & $\mathrm{Ru}$ & $\mathrm{Sw}$ & $\mathrm{Th}$ & $\mathrm{Tr}$ & $\mathrm{Ur}$ & $\mathrm{Vi}$ & $\mathrm{Zh}$ \\
\cline { 2 - 13 } & English & 0.90 & 0.55 & 0.61 & 0.69 & 0.60 & 0.76 & 0.88 & $\mathbf{0 . 4 7}$ & 0.66 & $\mathbf{0 . 4 4}$ & 0.56 & $\mathbf{0 . 3 5}$ & 0.60 & $\mathbf{0 . 4 2}$ & 0.70 \\
& Target & - & 0.77 & 0.88 & 0.82 & 0.89 & 0.78 & 0.87 & 0.90 & 0.85 & 0.87 & 0.93 & 0.85 & 0.93 & 0.93 & 0.93 \\
\hline
\end{tabular}

Table 3: Frequency of directional agreement between dev and test accuracy on MLDoc and XNLI (higher is better). We expect dev and test accuracy to generally increase and decrease together between randomly sampled checkpoints, which happens when using the target language dev set, but not when using the English dev set. English dev accuracy can be worse than random chance (50\%) at tracking target test accuracy; these values are bolded.

and Japanese (Ja) accuracies swing by several percentage points as training proceeds.

Indeed, for all of the MLDoc languages and for 7 of the 14 XNLI languages, the variation across 10 runs exceeds $2.5 \%$ (absolute), even though the En dev accuracy only varies within a narrow $0.9 \%$ range. To put this variation in context, we note that the MLDoc and XNLI test sets are relatively large, so a $2.5 \%$ difference in accuracy would be statistically significant (at the 5\% significance level using the usual test of proportions), which means that a paper claiming 'state-of-the-art' performance on these zero-shot tasks may be reporting strong results because of the large between-run variation, rather than a genuine improvement due to their proposed technique.

In other words, using the En dev accuracy for choosing the final model for zero-shot transfer leads to inconsistent results across different runs. The En dev accuracy is similar for each of our independent experiments, but the target test accuracy for each experiment fluctuates in a wide band.

\section{English dev accuracy and its relationship with zero-shot accuracy}

Experimenters use the En dev set for model selection under the assumption that zero-shot performance improves as En dev performance improves. We show that this assumption is often false.

We compare the abilities of En dev and target dev to predict directional changes in target test accuracy. In Table 3, we report the frequency of directional agreement on MLDoc and XNLI; i.e., how often does En dev accuracy increase/decrease in tandem with target test accuracy?

We randomly sample pairs of checkpoints where the change in target test accuracy for the pair is at least $0.5 \%$, and compute the proportion for which the En dev accuracy changed in the same direction. Table 3 shows that for MLDoc, En dev is not much better than a coin flip $(\sim 50 \%)$ at predicting the direction of the change in target test accuracy, while target dev tracks target test accuracy $90+\%$ of the time. For XNLI, En dev sometimes approaches target dev in predictive power (i.e., Es and Fr), but can fall short for other languages. In general, we see higher directional agreement in XNLI than 


\begin{tabular}{|c|c|c|c|c|c|c|c|c|c|c|c|c|c|c|c|c|}
\hline \multicolumn{2}{|l|}{ XNLI } & En & $\mathrm{Ar}$ & $\mathrm{Bg}$ & De & $\mathrm{El}$ & Es & $\mathrm{Fr}$ & $\mathrm{Hi}$ & $\mathrm{Ru}$ & $\mathrm{Sw}$ & Th & $\operatorname{Tr}$ & Ur & $\mathrm{Vi}$ & $\mathrm{Zh}$ \\
\hline \multicolumn{2}{|l|}{ Hu et al. (2020) } & 80.8 & 64.3 & 68.0 & 70.0 & 65.3 & 73.5 & 73.4 & 58.9 & 67.8 & 49.7 & 54.1 & 60.9 & 57.2 & 69.3 & 67.8 \\
\hline \multicolumn{2}{|l|}{ Nooralahzadeh et al. (2020) } & 81.4 & 64.6 & 67.8 & 69.7 & 65.7 & 73.9 & 73.5 & 58.6 & 67.9 & 47.6 & 52.5 & 59.0 & 58.7 & 70.1 & 68.9 \\
\hline \multicolumn{2}{|c|}{ Wu and Dredze (2019), Liang et al. (2020) } & 82.1 & 64.9 & 68.9 & 71.1 & 66.4 & 74.3 & 73.8 & 60.0 & 69.0 & 50.4 & 55.8 & 61.6 & 58.0 & 69.5 & 69.3 \\
\hline \multicolumn{2}{|l|}{ mBERT $\Delta$} & 1.3 & 0.6 & 1.1 & 1.4 & 1.1 & 0.8 & 0.4 & 0.4 & 1.2 & 2.8 & 3.3 & 2.6 & 1.5 & 0.8 & 1.5 \\
\hline \multirow{3}{*}{\multicolumn{2}{|c|}{$\begin{array}{l}\text { Hu et al. (2020) } \\
\text { Conneau et al. (2020)* } \\
\text { Phang et al. (2020) }\end{array}$}} & 88.7 & 77.2 & 83.0 & 82.5 & 80.8 & 83.7 & 82.2 & 75.6 & 79.1 & 71.2 & 77.4 & 78.0 & 71.7 & 79.3 & $\overline{78.2}$ \\
\hline & & 89.1 & 79.8 & 84.0 & 83.9 & 82.9 & 85.1 & 84.1 & 76.9 & 81.2 & 73.9 & 78.1 & 79.6 & 73.8 & 80.8 & 80.2 \\
\hline & & 89.3 & 79.8 & 82.7 & 83.8 & 81.3 & 84.4 & 83.7 & 77.3 & 79.2 & 72.4 & 77.1 & 78.9 & 72.6 & 80.0 & 79.6 \\
\hline \multicolumn{2}{|l|}{ XLM-R large $\Delta$} & 0.6 & 2.6 & 1.3 & 1.4 & 2.1 & 1.4 & 1.9 & 1.7 & 2.1 & 2.7 & 1.0 & 1.6 & 2.1 & 1.5 & 2.0 \\
\hline MLQA & En & $\mathrm{Ar}$ & & & $\mathrm{De}$ & & Es & is & & $\mathrm{Hi}$ & & $\overrightarrow{V_{i}}$ & Ti & & $\mathrm{Zh}$ & \\
\hline Lewis et a & 77.7 & 45 . & & & 57.9 & & 64. & .3 & & 43.8 & & 57. & .1 & & 57.5 & \\
\hline Hu et al. (2020) & 80.2 & 52. & & & 59.0 & & 67. & .4 & & 50.2 & & 61. & .2 & & 59.6 & \\
\hline Liang et al. (2020) & 80.5 & 50. & & & 63.8 & & 67. & .1 & & 47.9 & & 59. & .5 & & 55.4 & \\
\hline mBERT $\Delta$ & 2.8 & 6.6 & & & 5.9 & & 3.1 & 1 & & 6.4 & & 4. & 1 & & 4.2 & \\
\hline Conneau et al. (2020)* & $80.6 / 67.8$ & $63.1 /$ & 43.5 & & $.5 / 53$ & & $74.1 /$ & 56.0 & & $.2 / 51$ & & $71.3 /$ & 50.9 & & $8.0 / 4$ & $\overline{15.4}$ \\
\hline Phang et al. (2020) & $81.6 / 68.6$ & $62.7 /$ & 42.4 & & $1 / 52$ & & 72.21 & 53.0 & & $.0 / 50$ & & $69.5 /$ & 47.6 & & $7.9 / 4$ & \\
\hline Hu et al. (2020) & $83.5 / 70.6$ & $66.6 /$ & 47.1 & & $.1 / 54$ & & $74.1 /$ & 56.6 & & $.6 / 53$ & & $74.0 /$ & 52.9 & & $2.1 / 3$ & 37.0 \\
\hline XLM-R large $\Delta$ & $2.9 / 2.8$ & $3.9 /$ & & & $.6 / 2.9$ & & $1.9 /$ & 3.6 & & $6 / 2$ & & $4.5 /$ & 5.3 & & $5.9 / 8$. & \\
\hline
\end{tabular}

Table 4: Published zero-shot accuracies for mBERT and XLM-R large on XNLI, and published zero-shot F1 scores for mBERT and F1 / EM scores for XLM-R large on MLQA. * Conneau et al. (2020) tune on all dev sets jointly.

MLDoc, which we attribute to XNLI's target test sets being professional translations of En test.

Remarkably, for some languages (i.e., Ja in MLDoc and $\mathrm{Hi}, \mathrm{Sw}, \mathrm{Tr}$, and Vi in XNLI), the frequency of directional agreement is less than $50 \%$, which means that, more often than not, when En dev accuracy increases, target test accuracy for these languages decreases; we discuss this in Section 5. Since En dev accuracy does not reliably move in the same direction as target test accuracy, it is an inadequate metric for tracking zero-shot crosslingual transfer performance.

\section{Catastrophic forgetting}

The strange phenomenon in Table 3 (where the probability of directional agreement is sometimes less than 50\%) occurs even on XNLI, where the dev and test sets are translated from English and therefore have the same semantic content. We believe this phenomenon is a form of catastrophic forgetting (Kirkpatrick et al., 2017), where mBERT loses some cross-lingual knowledge from pre-training because it is fine-tuned on En data only.

In Figure 1, we plotted the En dev accuracy and the target test accuracy over time, for the language with the highest directional agreement (Es, 0.59) and for the language with the lowest directional agreement (Ja, 0.42) for MLDoc (see Table 3). From the figure, Es test accuracy does increase with En dev accuracy, while Ja test accuracy decreases as En dev accuracy increases. The same pattern holds with XNLI for Tr and En (not shown), where Turkish accuracy decreases somewhat as fine-tuning with English training data continues.

We conclude that En dev accuracy cannot detect when mBERT is improving on the En training data at the expense of non-En languages, and should not (solely) be used to assess zero-shot performance.

\section{Published results}

At the time of writing, over 70 papers have been published on the MLDoc and XNLI corpora. Several new datasets (e.g., Liu et al., 2019; Artetxe et al., 2020; Yang et al., 2019) for zero-shot crosslingual evaluation have been released and aggregated into benchmark suites like XGLUE (Liang et al., 2020) and XTREME (Hu et al., 2020). There are also more recent multilingual contextual embeddings (e.g., XLM-R large; Conneau et al., 2020) which is trained on significantly more data (2.5 terabytes). Notably, Conneau et al. (2020) uses the target dev sets jointly for checkpoint selection.

As with Table 1 for MLDoc, we search for signs of the variability found in our experimental setup across the zero-shot cross-lingual literature. In XNLI (Table 4) we see less drastic variations in $\Delta$. However, for variations in En of 0.6 (XLMR) and 1.3 (mBERT) points, we see variations of 


\begin{tabular}{lccccccc}
\hline MLDoc & De & Es & Fr & It & Ja & Ru & Zh \\
\hline Target dev (oracle) & $\mathbf{8 9 . 7}$ & $\mathbf{8 4 . 4}$ & $\mathbf{8 4 . 4}$ & $\mathbf{7 3 . 1}$ & $\mathbf{7 5 . 5}$ & $\mathbf{7 7 . 1}$ & $\mathbf{8 1 . 1}$ \\
Best En dev & 87.6 & 82.3 & 78.4 & 69.2 & 67.7 & 71.7 & 70.2 \\
Best published & 82.4 & 79.5 & 83.0 & 68.9 & 73.2 & 73.7 & 76.9 \\
\hline
\end{tabular}

\begin{tabular}{lcccccccccccccc}
\hline XNLI & $\mathrm{Ar}$ & $\mathrm{Bg}$ & $\mathrm{De}$ & $\mathrm{El}$ & $\mathrm{Es}$ & $\mathrm{Fr}$ & $\mathrm{Hi}$ & $\mathrm{Ru}$ & $\mathrm{Sw}$ & $\mathrm{Th}$ & $\mathrm{Tr}$ & $\mathrm{Ur}$ & $\mathrm{Vi}$ & $\mathrm{Zh}$ \\
\hline Target dev (oracle) & $\mathbf{6 6 . 5}$ & $\mathbf{7 0 . 0}$ & $\mathbf{7 2 . 0}$ & $\mathbf{6 7 . 8}$ & $\mathbf{7 5 . 9}$ & $\mathbf{7 4 . 6}$ & $\mathbf{6 3 . 2}$ & $\mathbf{7 0 . 7}$ & $\mathbf{5 2 . 9}$ & $\mathbf{5 7 . 3}$ & $\mathbf{6 3 . 0}$ & $\mathbf{6 0 . 5}$ & $\mathbf{7 1 . 4}$ & $\mathbf{7 1 . 3}$ \\
Best En dev & 66.0 & 69.7 & 71.8 & 67.6 & 75.8 & 74.6 & 61.7 & 69.6 & 50.9 & 55.3 & 61.9 & 60.2 & 71.3 & 71.3 \\
Best published & 64.9 & 68.9 & 71.1 & 66.4 & 74.3 & 73.8 & 60.0 & 69.0 & 50.4 & 55.8 & 61.6 & 58.7 & 70.1 & 69.3 \\
\hline
\end{tabular}

Table 5: Oracle zero-shot accuracies with mBERT across 10 independent runs, using target dev to select the best checkpoint for each language. This provides an upper bound on the achievable zero-shot accuracy. Published results are derived from sources in Tables 1, 4, and 6. Best En dev results are from Table 2.

$\geq 2.5$ points in linguistically distant languages (Ar, $\mathrm{Sw}, \mathrm{Th}, \mathrm{Tr})$, languages which agreed with En dev $\leq 56 \%$ during finetuning (Table 3 ). We also include MLQA (Lewis et al., 2020), where a variation of 2.8 in En mBERT results gave even higher variations ( $\geq 4.0$ points) in all languages except Es. A similar effect occurs with XLM-R and the more distant languages (Ar, Vi, Zh). In particularly, Chinese ( $\mathrm{Zh}$ ) degrades by 5.9 points as En improves, which may be evidence of directional disagreement in MLQA between En dev and $\mathrm{Zh}$ test.

In Table 6, results on CoNLL 2002/2003 (Sang and De Meulder, 2003) show slightly increased variation relative to En. We also include the remaining MLDoc languages for completeness. ${ }^{3}$

\begin{tabular}{lcccc}
\hline CoNLL 2002/2003 & En & De & Es & Nl \\
\hline Liang et al. (2020) & 90.6 & 69.2 & 75.4 & 77.9 \\
Pires et al. (2019) & 90.7 & 69.7 & 73.6 & 77.4 \\
Keung et al. (2019) & 91.1 & 68.6 & 75.0 & 77.5 \\
Bari et al. (2020) & 91.1 & 71.0 & 74.8 & 79.6 \\
Wu and Dredze (2019) & 92.0 & 69.6 & 75.0 & 77.6 \\
\hline mBERT $\Delta$ & 1.4 & 2.4 & 1.8 & 2.2 \\
\hline & & & & \\
\hline MLDoc (cont.) & En & De & Es & It \\
\hline Eisenschlos et al. (2019) & 93.2 & 82.4 & 75.0 & 68.3 \\
Dong and de Melo (2019) & 94.2 & 78.9 & 79.5 & 68.7 \\
Keung et al. (2019) & 94.2 & 79.8 & 72.1 & 63.7 \\
Wu and Dredze (2019) & 94.2 & 80.2 & 72.6 & 68.9 \\
\hline mBERT $\Delta$ & 1.0 & 3.5 & 7.4 & 5.2 \\
\hline
\end{tabular}

Table 6: Published zero-shot F1 scores for mBERT on cross-lingual NER (CoNLL 2002/2003), and published zero-shot accuracies for mBERT on MLDoc for languages not included in Table 1.

\footnotetext{
${ }^{3}$ The English result from Eisenschlos et al. (2019) is from b7e3a5 of https://github.com/n-waves/multifit.
}

\section{Recommendations and discussion}

Using a poor metric like En dev accuracy to select a model checkpoint is similar to picking a checkpoint at random. This would not be a major issue if the variance between different training runs were low; the test performance would, in that case, be consistently mediocre. The problem arises when the variability is high, which we have seen experimentally (Table 2) and in the wild (Table 1).

We showed that independent experiments can report very different results, which prevents us from making meaningful comparisons between different baselines and methods. Currently, it is standard practice to use the En dev accuracy for checkpoint selection in the zero-shot cross-lingual setting. However, we showed that using En dev accuracy for checkpoint selection leads to somewhat arbitrary zero-shot results.

Therefore, we propose reporting oracle accuracies, where one still fine-tunes using English data, but selects a checkpoint using target dev. This represents the maximum achievable zero-shot accuracy. Note that we do not use target dev for hyperparameter tuning; we are using target dev to avoid selecting bad checkpoints within each fine-tuning experiment. Table 5 shows our oracle results on MLDoc and XNLI. Reporting this upper bound makes results more consistent by avoiding arbitrarily bad checkpoints. To avoid unexpected variation in future cross-lingual publications, we recommend that authors report oracle accuracies alongside their zero-shot results.

\section{References}

Mikel Artetxe, Gorka Labaka, Eneko Agirre, and Kyunghyun Cho. 2018. Unsupervised neural machine translation. In ICLR. 
Mikel Artetxe, Sebastian Ruder, and Dani Yogatama. 2020. On the cross-lingual transferability of monolingual representations. In $A C L$, pages 4623-4637.

M Saiful Bari, Muhammad Tasnim Mohiuddin, and Shafiq Joty. 2020. MultiMix: A robust data augmentation strategy for cross-lingual NLP. arXiv preprint arXiv:2004.13240.

Alexis Conneau, Kartikay Khandelwal, Naman Goyal, Vishrav Chaudhary, Guillaume Wenzek, Francisco Guzmán, Edouard Grave, Myle Ott, Luke Zettlemoyer, and Veselin Stoyanov. 2020. Unsupervised cross-lingual representation learning at scale. In $A C L$, pages $8440-8451$.

Alexis Conneau and Guillaume Lample. 2019. Crosslingual language model pretraining. In NeurIPS, pages 7057-7067.

Alexis Conneau, Guillaume Lample, Ruty Rinott, Adina Williams, Samuel R Bowman, Holger Schwenk, and Veselin Stoyanov. 2018. XNLI: Evaluating cross-lingual sentence representations. In EMNLP, pages 2475-2485.

Jacob Devlin, Ming-Wei Chang, Kenton Lee, and Kristina Toutanova. 2019. BERT: Pre-training of deep bidirectional transformers for language understanding. In NAACL-HLT, pages 4171-4186.

Xin Luna Dong and Gerard de Melo. 2019. A robust self-learning framework for cross-lingual text classification. In EMNLP-IJCNLP, pages 6307-6311.

Julian Eisenschlos, Sebastian Ruder, Piotr Czapla, Marcin Kardas, Sylvain Gugger, and Jeremy Howard. 2019. MultiFiT: Efficient multi-lingual language model fine-tuning. In EMNLP-IJCNLP, pages 5701-5706.

Jian Guo, He He, Tong He, Leonard Lausen, $\mathrm{Mu} \mathrm{Li}$, Haibin Lin, Xingjian Shi, Chenguang Wang, Junyuan Xie, Sheng Zha, Aston Zhang, Hang Zhang, Zhi Zhang, Zhongyue Zhang, Shuai Zheng, and Yi Zhu. 2020. GluonCV and GluonNLP: Deep learning in computer vision and natural language processing. JMLR, 21(23):1-7.

Junjie Hu, Sebastian Ruder, Aditya Siddhant, Graham Neubig, Orhan Firat, and Melvin Johnson. 2020. XTREME: A massively multilingual multitask benchmark for evaluating cross-lingual generalization. In ICML.

Phillip Keung, Yichao Lu, and Vikas Bhardwaj. 2019. Adversarial learning with contextual embeddings for zero-resource cross-lingual classification and NER. In EMNLP-IJCNLP, pages 1355-1360.

James Kirkpatrick, Razvan Pascanu, Neil Rabinowitz, Joel Veness, Guillaume Desjardins, Andrei A Rusu, Kieran Milan, John Quan, Tiago Ramalho, Agnieszka Grabska-Barwinska, et al. 2017. Overcoming catastrophic forgetting in neural networks. PNAS, 114(13):3521-3526.
Guillaume Lample, Alexis Conneau, Ludovic Denoyer, and Marc'Aurelio Ranzato. 2018a. Unsupervised machine translation using monolingual corpora only. In ICLR.

Guillaume Lample, Alexis Conneau, Marc'Aurelio Ranzato, Ludovic Denoyer, and Hervé Jégou. 2018b. Word translation without parallel data. In ICLR.

Patrick Lewis, Barlas Oğuz, Ruty Rinott, Sebastian Riedel, and Holger Schwenk. 2020. MLQA: Evaluating cross-lingual extractive question answering. In $A C L$, pages $7315-7330$.

Yaobo Liang, Nan Duan, Yeyun Gong, Ning Wu, Fenfei Guo, Weizhen Qi, Ming Gong, Linjun Shou, Daxin Jiang, Guihong Cao, et al. 2020. XGLUE: A new benchmark dataset for cross-lingual pretraining, understanding and generation. arXiv preprint arXiv:2004.01401.

Jiahua Liu, Yankai Lin, Zhiyuan Liu, and Maosong Sun. 2019. XQA: A cross-lingual open-domain question answering dataset. In $A C L$, pages 2358 2368.

Gábor Melis, Chris Dyer, and Phil Blunsom. 2018. On the state of the art of evaluation in neural language models. In ICLR.

Farhad Nooralahzadeh, Giannis Bekoulis, Johannes Bjerva, and Isabelle Augenstein. 2020. Zero-shot cross-lingual transfer with meta learning. arXiv preprint arXiv:2003.02739.

Jason Phang, Phu Mon Htut, Yada Pruksachatkun, Haokun Liu, Clara Vania, Katharina Kann, Iacer Calixto, and Samuel R. Bowman. 2020. English intermediate-task training improves zeroshot cross-lingual transfer too. arXiv preprint arXiv:2005.13013.

Telmo Pires, Eva Schlinger, and Dan Garrette. 2019. How multilingual is Multilingual BERT? In $A C L$, pages 4996-5001.

Erik F Sang and Fien De Meulder. 2003. Introduction to the CoNLL-2003 shared task: Languageindependent named entity recognition. In CoNLL, pages 142-147.

Holger Schwenk and Xian Li. 2018. A corpus for multilingual document classification in eight languages. In $L R E C$, pages 3548-3551.

Shijie Wu and Mark Dredze. 2019. Beto, Bentz, Becas: The surprising cross-lingual effectiveness of BERT. In EMNLP-IJCNLP, pages 833-844.

Yinfei Yang, Yuan Zhang, Chris Tar, and Jason Baldridge. 2019. PAWS-X: A cross-lingual adversarial dataset for paraphrase identification. In EMNLP-IJCNLP, pages 3685-3690. 